\title{
Pointing in a different direction: a case of bilateral absence of extensor indicis
}

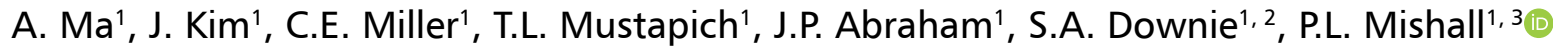 \\ ${ }^{1}$ Department of Anatomy and Structural Biology, Albert Einstein College of Medicine, Bronx, NY, United States \\ ${ }^{2}$ Department of Physical Medicine and Rehabilitation, Albert Einstein College of Medicine, Bronx, NY, United States \\ ${ }^{3}$ Department of Ophthalmology and Visual Sciences, Albert Einstein College of Medicine, Bronx, NY, United States
}

[Received: 5 November 2020; Accepted: 5 March 2021; Early publication date: 22 March 2021]

\begin{abstract}
Understanding anatomical variations as well as normal anatomy of the muscles and tendons of the hand is vital for successful clinical evaluation and surgery. A number of extensor muscle and tendon variations have been reported in the literature, including duplication, triplication, and absence. We report a rare anatomical variation that includes bilateral absence of the extensor indicis (EI) muscles and bilateral duplication of the extensor digitorum (ED) tendon to the second digit in the forearm of an 83-year-old male cadaver during routine upper limbs dissection. In the present case, only three muscles were present in the deep compartment: extensor pollicis longus (EPL), extensor pollicis brevis (EPB), and abductor pollicis longus (APL) with bilateral absence of El. The reported prevalence of bilateral absence of $E I$ muscle and tendon ranges from $0.5 \%$ to $3.5 \%$. The prevalence of an additional index tendon arising bilaterally from the ED muscle belly is $3.2 \%$ of the population. Extension of the index finger is governed by the actions of EI and ED. However, the four tendons of ED are linked to each other by juncturae tendinum, restricting independent extension of the digits in certain postures, e.g. when the hand is fisted. With fisted hand, El controls extension of the index finger. Clinically, El tendons are used for tendon reconstruction procedures to restore function to the hand and thumb after trauma or tendon rupture. This report highlights the importance of anticipating anatomical variations and conducting pre-operative evaluations to confirm the presence of El when planning tendon transfer procedures. (Folia Morphol 2022; 81, 2: 520-525)
\end{abstract}

Key words: extensor indicis, forearm, congenital, index finger, hand

\section{INTRODUCTION}

Thorough knowledge of the arrangement of tendons on the dorsum of the hand is essential when performing surgical procedures for tendon repair or tendon transfer within the hand. The extensor muscle-tendon units serving the second digit include extensor indicis (EI) and extensor digitorum (ED). EI attaches proximally to the posterior surface of the distal third of the ulna and interosseous membrane and passes distally through the fourth dorsal compartment of the wrist together with ED tendons. The El tendon travels on the ulnar side of the ED index

Address for correspondence: P.L. Mishall, Ass. Prof., Departments of Anatomy and Structural Biology and Ophthalmology and Visual Sciences, Albert Einstein College of Medicine, 1300 Morris Park Ave, F620SD, Bronx, NY 10461, USA, tel: +1 (718) 430-3423, fax: +1 (718) 430-8996, e-mail: priti.mishall@eisnteinmed.org

This article is available in open access under Creative Common Attribution-Non-Commercial-No Derivatives 4.0 International (CC BY-NC-ND 4.0) license, allowing to download articles and share them with others as long as they credit the authors and the publisher, but without permission to change them in any way or use them commercially. 
tendon to insert into the extensor expansion of the second digit [4]. Typically, El produces extension of the second digit at the metacarpophalangeal, proximal and distal interphalangeal joints independent of the ED index tendon $[9,12]$. It serves this function regardless of hand posture.

The four tendons of ED arise proximally from a common muscle belly attached to the lateral epicondyle of the humerus, and insert distally into the extensor hoods on the dorsum of the second through fifth metacarpal heads [8]. Just proximal to the metacarpophalangeal (MCP) joints, the ED tendons are joined to each other by oblique connective tissue bands, juncturae tendinum (JT) [6]. ED produces extension of the digits primarily at the MCP joints and secondarily at the interphalangeal joints. However, due to the JT, digital extension is restricted in certain postures such as fisted hand.

Instances of El variants, including multiple tendon slips $[9,14,15]$ or absence of the tendon $[8,10,18]$, have been noted in the literature. Absence of the $\mathrm{EI}$ muscle-tendon unit is very uncommon with a reported prevalence of $0.5-3.5 \%$ in previous meta-analyses $[8,25]$. Only 2 case reports describe the absence of EI bilaterally $[22,26]$. Variations of the ED attachment to the second digit are also uncommon, with double slip tendon variants observed at a prevalence of $3.2 \%$ [1].

Extensor indicis tendons are commonly used in tendon transfer procedures to reconstruct the abductor pollicis longus (APL) or extensor pollicis longus (EPL) tendons. Because the index finger receives the ED tendon as well as the El tendon, it should maintain the ability to extend if the El tendon is removed [11, 13]. It is critical, therefore, to be aware of variations of the El muscle, especially those that involve absence of its tendon, to evaluate patients prior to tendon transfer surgery $[16,21]$. We report a case of bilateral absence of the El muscle-tendon unit and bilateral presence of an additional tendon slip arising from ED to the second digit.

\section{CASE REPORT}

During routine dissection of an 83-year-old male cadaver at the Albert Einstein College of Medicine, Bronx, NY, the extensor compartments of the forearms and dorsum of the hands were dissected by removing the skin and fascia to visualise the underlying muscles. The extensor muscles were identified and their tendons cleaned and followed to their distal attachments. Both the muscle belly and tendon of the El were absent bilaterally. And, bilaterally, an additional tendon slip arose from the ED muscle belly and inserted at the second digit (Figs. 1B, C). The presumptive normal tendon of ED to the second digit was connected with the other ED tendons via JT. On close observation, the JT were thin fascia-like tissues, as opposed to dense fibrous tissues. This quality confers a wider range of mobility for independent digital extension. No surgical incisions were found on either hand or forearm, suggesting that no surgical procedures had been performed. Further inspection of the extensor compartment showed only three muscles arising from the posterior surface of the radius, ulna, and interosseous membrane (Figs. $2 \mathrm{~B}, \mathrm{C})$. These three muscles were the EPL, extensor pollicis brevis (EPB), and APL. Also, normal right hand with $\mathrm{El}$ is shown (Figs. 1A, 2A).

\section{DISCUSSION}

The normal presentation of $\mathrm{El}$ is one muscle belly attached to the posterior surface of the ulna and interosseous membrane with one tendon descending to the extensor aponeurosis of the second digit. The El tendon typically runs deep, ulnar and parallel to the ED index tendon. Studies of the dorsum of the hand indicate that variations in tendons to the second digit are rare $[1,6]$. An extensive literature review revealed only 2 case reports of bilateral absence of the El similar to our case $[22,26]$. Additionally, Bergman's Comprehensive Encyclopedia of Human Anatomic Variation reports that muscles of the index finger are rarely absent; however, the authors do not indicate the prevalence of absent El [2]. An early study of 263 upper extremities did not report absent El [5]. However, in the same study Cauldwell et al. [5] referenced 5 cases of absent El muscle that were published between 1806 and 1936. It was not indicated whether these were unilateral or bilateral. Hence, the current case of bilateral complete absence of $\mathrm{El}$ is rare.

Furthermore, the current case is unique due to the presence of bilateral additional tendon slips arising from the ED muscle belly to the second digit. A thorough literature review indicated that variations, in the form of additional tendons to the index finger, were observed in two categories. In the first category, the additional tendon did not arise from ED but arose as a separate muscle either from the radius $[19,24]$ or the ulna [10]. In the second category, the additional tendon to the index finger arose from ED [3]. A dissection-based study of 13 cadavers found $2(7.69 \%)$ cases 


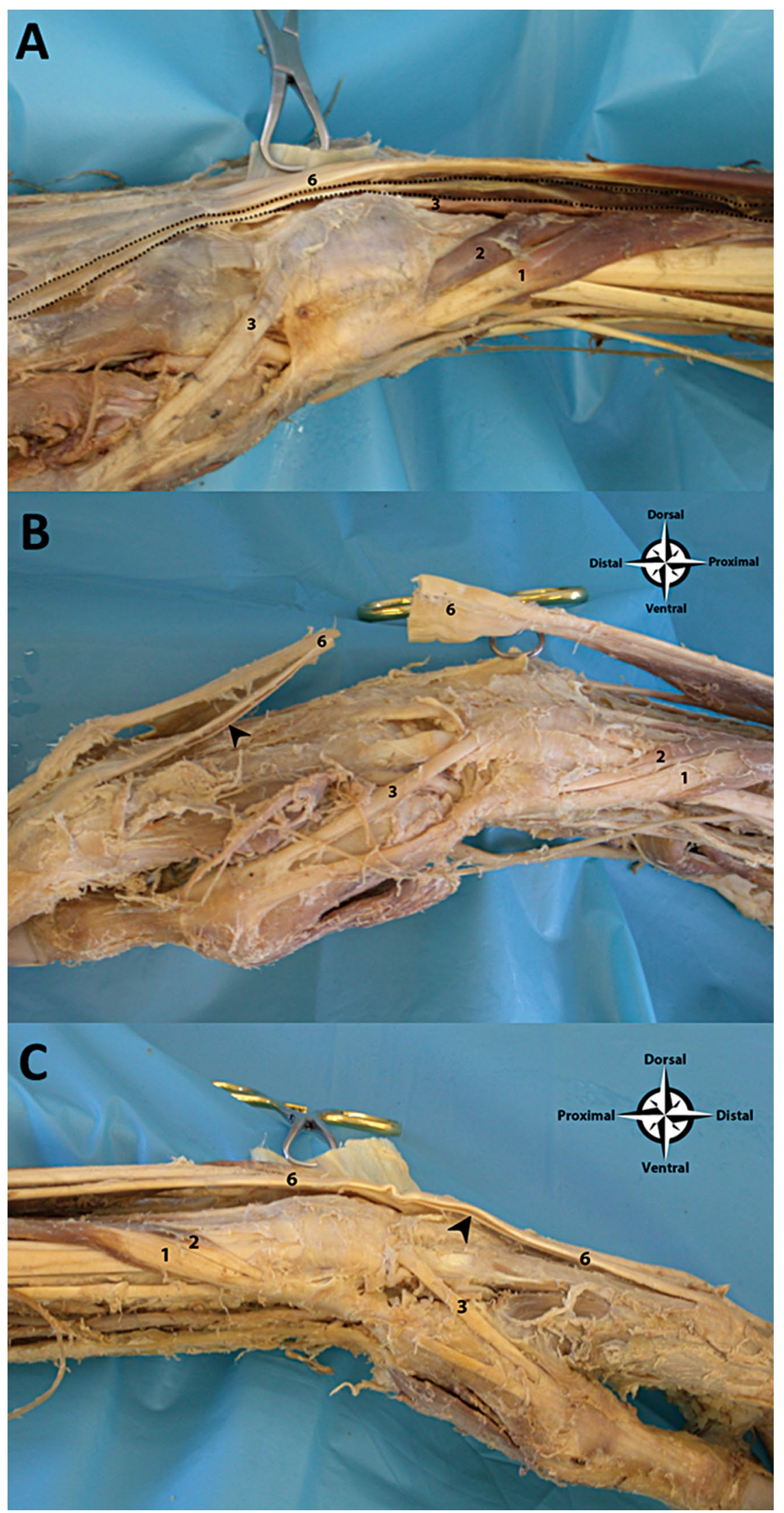

Figure 1. A. Normal right hand with extensor indicis present (dotted line); B, C. Right and left hands, respectively, with additional tendinous slip (arrowhead) arising from extensor digitorum; 1 - abductor pollicis longus; 2 - extensor pollicis brevis; 3 - extensor pollicis longus; 6 - extensor digitorum. 


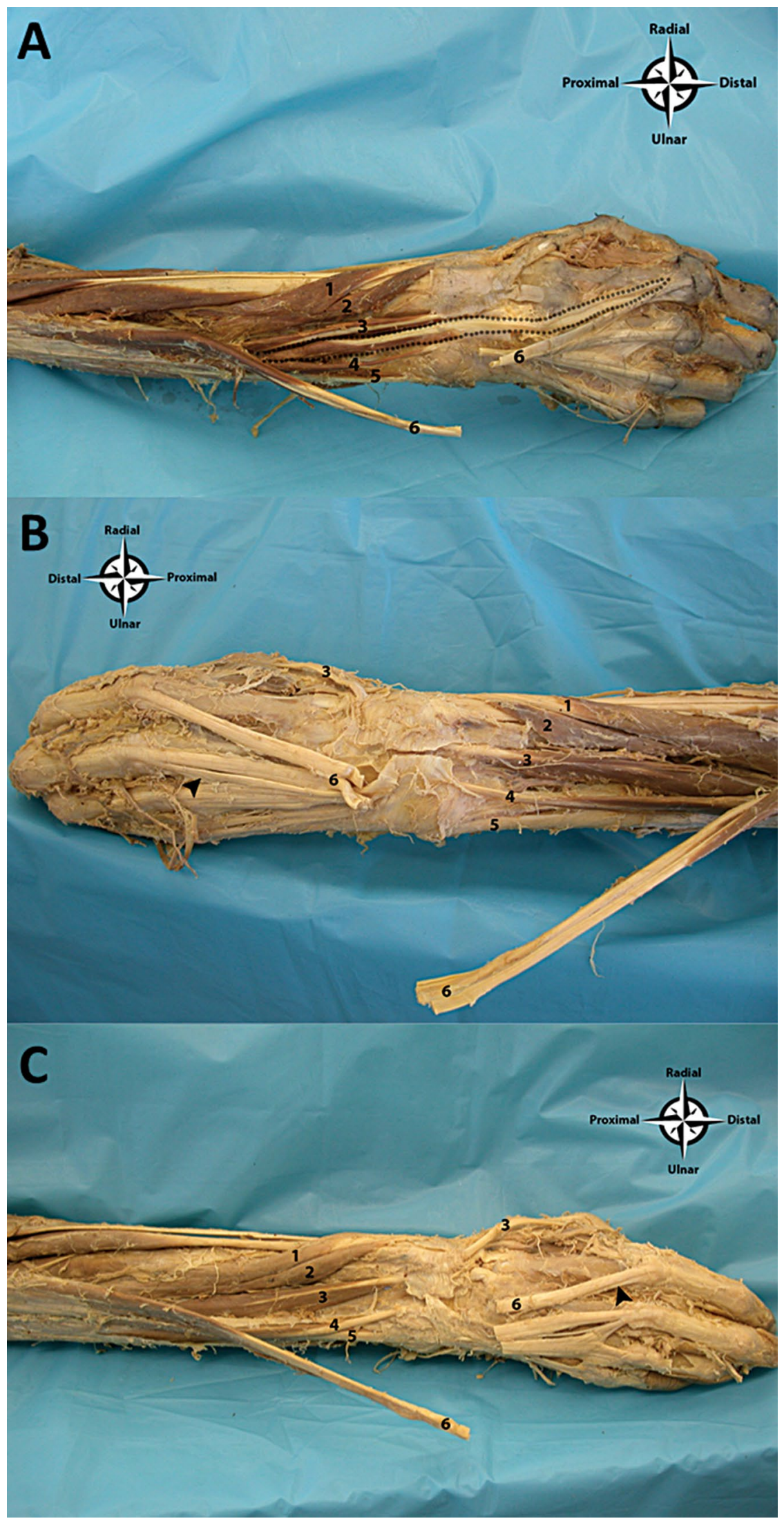

Figure 2. A. Normal right hand with extensor indicis present (dotted line); B, C. Left and right hands, respectively, with absent extensor indices. Additional tendinous slip arising from extensor digitorum indicated by arrowhead; 1 - abductor pollicis longus; 2 - extensor pollicis brevis; 3 - extensor pollicis longus; 4 - extensor digiti minimi; 5 - extensor carpi ulnaris; 6 - extensor digitorum (reflected). 
in which the ED tendon to the index finger originated as two tendons that fused before reaching the MCP joint and inserted into the extensor expansion of the index finger as a single tendon [1]. The study conducted by Abdel-Hamid et al. [1], on 95 upper limbs, reported $3(3.2 \%)$ cases of double tendons arising from the ED going to the index finger. In the current case, the ED gave two tendons that inserted at the index finger as two tendons. The other three ED tendons, one to each digit 3-5, were typical. Another dissection-based study of 54 upper limbs did not report double tendons that arose from ED to index finger [6].

In summary, the findings of bilateral absence of the El muscle-tendon $[22,26]$ and additional slip of ED tendon to index finger have been reported as separate occurrences. The current case is unique in having both variations - bilateral absence of the El muscle-tendon unit with the presence of bilateral additional tendon slip from ED that inserted onto the index finger.

The functional test for the presence of $\mathrm{El}$ is independent extension of the index finger with the hand fisted. In the complete absence of El, ED is responsible for extending the index finger. However, the four ED tendons are connected to each other by JT that limit independent extension of the digits. Von Schroeder et al. [23] described three types of JTs: type 1 (thin, fascia-like), type 2 (thicker, fibrous), and type 3 (thickest, tendinous band). Yammine [24] reported the prevalence of $\mathrm{JT}$ between the index finger and middle finger as type $1(95 \%)$, type $2(5 \%)$, and type $3(0 \%)$. Furthermore, they showed that type 1 JTs accommodated independent extension of the four medial digits whereas type 2 and 3 JTs were restrictive [24]. In the present case, we observed thin fascia-like JTs similar to that described as type 1. We speculate that the individual, when living, may have had the ability to independently extend the index fingers in the absence of El.

It is most likely that variations in limb musculoskeletal development resulted in the bilateral absence of the El muscle-tendon unit and the bilateral presence of additional tendon slips arising from the ED muscle bellies. During embryological development the extensor muscle mass of the forearm differentiates into three sections: radial, superficial and deep [17]. The radial section gives rise to brachioradialis, and extensor carpi radialis longus and brevis. The remaining portion of muscle mass divides into superficial and deep sections. The superficial section differentiates into extensor carpi ulnaris, extensor digitorum, and extensor digiti minimi, and is highly evolutionarily conserved [23]. The deep section, which has undergone the most significant evolutionary variation, gives rise to the extensor indicis, abductor pollicis longus, and extensor pollicis longus and brevis [10]. These observations are consistent with the variations identified in the present case, although they do not address the bilateral versus unilateral nature of the anomalies.

Clinically, absence of the El tendon results in significant consequences, as it is the tendon most commonly used for reconstruction of the EPL and abductor pollicis longus (AbPL) tendons [16, 20]. Spontaneous rupture of the EPL tendon, occurring most frequently in patients with rheumatoid arthritis but also secondary to direct trauma and distal radial fracture, results in decreased capacity for extension at the interphalangeal and metacarpophalangeal joints of the thumb $[16,20]$. Tendon transfer of the El is the current gold standard for efficacious EPL reconstruction, although extension deficits of the index finger, as well as loss of pinch strength, have been noted following El resection [16]. Cases in which only one extensor tendon to the index finger can be located necessitate alternative sources of graft transfer, most commonly palmaris longus. Ultrasound, or other imaging techniques, are used to verify the presence of the El muscle-tendon unit in preparation for surgical reconstruction $[7,21]$.

\section{CONCLUSIONS}

This cadaveric case report documents bilateral absence of the El muscle-tendon unit and bilateral presence of additional tendon slips arising from the ED muscle, running parallel to the ED index tendon, inserting on the second digit. We hypothesize that variations in upper limb musculoskeletal development resulted in the rare bilateral absence of the El muscle-tendon unit and bilateral presence of an additional tendon slip to the second digit arising from ED muscle belly. To summarise, preservation of hand dynamics through surgical reconstruction requires anticipation of anatomical variations of the hand and verification of the presence of both index tendons in order to avoid potential significant complications.

\section{Acknowledgements}

The authors acknowledge and gratefully thank the individual whose body and tissues were used in this study for the advancement of physician education and patient care.

Conflict of interest: None declared 


\section{REFERENCES}

1. Abdel-Hamid GA, El-Beshbishy RA, Abdel Aal IH. Anatomical variations of the hand extensors. Folia Morphol. 2013; 72(3): 249-257, doi: 10.5603/fm.2013.0040, indexed in Pubmed: 24068687

2. Akita K, Nimura A. Bergman's Comprehensive Encyclopedia of Human Anatomic Variation. In: Tubbs RS, Shoja MM, Loukas M (eds.). Forearm muscles. Wiley Blackwell, New Jersey 2016: 298-314.

3. Arquez HF. An anatomical study of the musculus flexor digitorum brevis. Int Arch Med. 2017; 10, doi: 10.3823/2486.

4. Biant LC. Pectoral girdle and Upper limb. In: Gray's Anatomy: The Anatomical Basis of Clincial Practice. Elsevier, New York 2016: 837-861.

5. Cauldwell EW, Anson BJ, Wright RR. The extensor indicis proprius muscle - a study of 263 consecutive specimans. Q Bull Northwest Univ Med Sch. 1943; 17(4): 267-279.

6. Celik S, Bilge $O$, Pinar $Y$, et al. The anatomical variations of the extensor tendons to the dorsum of the hand. Clin Anat. 2008; 21(7): 652-659, doi: 10.1002/ca.20710, indexed in Pubmed: 18792963.

7. Dezfuli B, Taljanovic MS, Melville DM, et al. Accuracy of high-resolution ultrasonography in the detection of extensor tendon lacerations. Ann Plast Surg. 2016; 76(2): 187-192, doi: 10.1097/SAP.0000000000000524, indexed in Pubmed: 26101990.

8. El-Badawi MG, Butt MM, al-Zuhair AG, et al. Extensor tendons of the fingers: arrangement and variations - II. Clin Anat. 1995; 8(6): 391-398, doi: 10.1002/ca.980080604, indexed in Pubmed: 8713158.

9. Garbelotti JS, Yukio FT, Rodrigues PV, et al. Extensor indicis brevis Muscle: an Unusual Muscular Variant. Int J Morphol. 2012; 30(3): 1071-1073, doi: 10.4067/s071795022012000300050 .

10. Georgiev GP, Tubbs RS, Iliev A, et al. Extensor indicis proprius muscle and its variants together with the extensor digitorum brevis manus muscle: a common classification. Clinical significance in hand and reconstructive surgery. Surg Radiol Anat. 2018; 40(3): 271-280, doi: 10.1007/ s00276-018-1981-9, indexed in Pubmed: 29383419.

11. Gonzalez M, Weinzweig N, Kay T, et al. Anatomy of the extensor tendons to the index finger. J Hand Surg. 1996; 21(6): 988-991, doi: 10.1016/s0363-5023(96)80305-4.

12. Gonzalez M, Weinzweig N, Kay T, et al. Anatomy of the extensor tendons to the index finger. J Hand Surg. 1996; 21(6): 988-991, doi: 10.1016/s0363-5023(96)80305-4.

13. Kitano K, Tada K, Shibata $T$, et al. Independent index extension after indicis proprius transfer: Excision of juncturae tendinum. J Hand Surg. 1996; 21(6): 992-996, doi: 10.1016/s0363-5023(96)80306-6.

14. Li J, Ren ZF. Bilateral extensor indicis brevis: a rare muscular variant. Rom J Morphol Embryol. 2012; 53(1): 185-187, indexed in Pubmed: 22395520.
15. Li J, Ren ZF. Bilateral extensor medii proprius with split tendon of extensor indicis proprius, a rare anatomical variant. Rom J Morphol Embryol. 2013; 54(3): 639-641, indexed in Pubmed: 24068417.

16. Matsumae G, Motomiya M, Iwasaki N. Failed reconstruction of the extensor pollicis longus in a patient with a major variation of the extensor indicis proprius tendon: a case report. J Hand Surg Asian Pac Vol. 2018; 23(1): 132-136, doi: 10.1142/S2424835518720062, indexed in Pubmed: 29409420.

17. Nayak SR, Vadgaonkar R, Krishnamurthy A, et al. An anomalous muscle in the forearm extensor compartment. Clinics (Sao Paulo). 2009; 64(3): 262-263, doi: 10.1590/s180759322009000300019, indexed in Pubmed: 19330255.

18. Ogura T, Inoue H, Tanabe G. Anatomic and clinical studies of the extensor digitorum brevis manus. J Hand Surg. 1987; 12(1): 100-107, doi: 10.1016/s0363-5023(87)80171-5.

19. Ranade AV, Rai R, Prabhu LV, et al. Incidence of extensor digitorum brevis manus muscle. Hand (N Y). 2008; 3(4): 320-323, doi: 10.1007/s11552-008-9111-5, indexed in Pubmed: 18780016.

20. Schaller P, Baer W, Carl HD. Extensor indicis-transfer compared with palmaris longus transplantation in reconstruction of extensor pollicis longus tendon: a retrospective study. Scand J Plast Reconstr Surg Hand Surg. 2007; 41(1): 33-35, doi: 10.1080/02844310601070351, indexed in Pubmed: 17484183.

21. Taylor J, Casaletto JA. Absence of extensor indicis tendon complicating reconstruction of the extensor pollicis longus. J Hand Surg Eur Vol. 2017; 42(5): 528-529, doi: $10.1177 / 1753193416636609$, indexed in Pubmed: 26936748

22. Trivedi $S$, Siddiqui $A$, Sinha $T$, et al. Absence of extensor indicis: A rare anatomical variant. Int J Biomed Res. 2014; 5(1): 61 , doi: 10.7439/ijbr.v5i1.442.

23. von Schroeder HP, Botte M, Gellman H. Anatomy of the juncturae tendinum of the hand. J Hand Surg Am. 1990; 15(4): 595-602, doi: 10.1016/s0363-5023(09)90021-1.

24. Yammine K. The prevalence of the extensor indicis tendon and its variants: a systematic review and meta-analysis. Surg Radiol Anat. 2015; 37(3): 247-254, doi: 10.1007/ s00276-014-1352-0, indexed in Pubmed: 25096501.

25. Yoshida Y. Anatomical studies on the extensor pollicis et indicis accessorius muscle and the extensor indicis radialis muscle in Japanese. Okajimas Folia Anat Jpn. 1995; 71(6): 355-363, doi: 10.2535/ofaj1936.71.6_355, indexed in Pubmed: 7739845.

26. Zilber S, Oberlin C. Anatomical variations of the extensor tendons to the fingers over the dorsum of the hand: a study of 50 hands and a review of the literature. Plast Reconstr Surg. 2004; 113(1): 214-221, doi: 10.1097/01. PRS.0000091163.86851.9C, indexed in Pubmed: 14707639 . 